\title{
Highly Enantioselective Iridium-Catalyzed Hydrogenation of Heteroaromatic Compounds, Quinolines
}

\author{
Wen-Bo Wang, Sheng-Mei Lu, Peng-Yu Yang, Xiu-Wen Han, and Yong-Gui Zhou* \\ Dalian Institute of Chemical Physics, the Chinese Academy of Sciences, Dalian 116023, P. R. China
}

Received March 30, 2003; E-mail: ygzhou@dicp.ac.cn

Catalytic asymmetric hydrogenations of prochiral unsaturated compounds, such as olefin, ketone, and imine, have been intensively studied and are considered as a versatile method of creating a chiral carbon center. ${ }^{1}$ However, asymmetric hydrogenation of heteroaromatic compounds is less explored, resonance stability of heteroaromatic compounds might impede the enantioselective hydrogenation. $^{2}$ To date, only a few papers on homogeneous asymmetric hydrogenation of heteroaromatic compounds have been reported. Kuwano and co-workers reported a highly effective hydrogenation of $\mathrm{N}$-Boc- or Ac-substituted indoles by application of $\mathrm{Rh} / \mathrm{Ph}$-TRAP/Cs ${ }_{2} \mathrm{CO}_{3}$ catalyst, ${ }^{3}$ and up to $95 \%$ ee was obtained. Bianchini developed an orthometalated dihydride iridium complex for hydrogenation of 2-methylquinoxaline with $90 \%$ ee. ${ }^{4}$ Studer and co-workers employed $\mathrm{Rh}(\mathrm{NBD})_{2} \mathrm{BF}_{4} / \mathrm{DIOP}$ as the catalyst for hydrogenation of monosubstituted pyridine and furans with only $24-27 \%$ ee. ${ }^{5 a} \mathrm{Rh}(\mathrm{DIOP}) \mathrm{H}$ was used as the catalyst for hydrogenation of 2-methylquinoxaline with only $3 \%$ ee. ${ }^{5 \mathrm{~b}}$ The search for a new catalytic system for asymmetric hydrogenation of heteroaromatic compounds is still a challenge.

Direct hydrogenation of easily available quinoline derivatives is the most convenient route to synthesize tetrahydroquinoline derivatives, which are important organic synthetic intermediates ${ }^{6}$ and structural units of alkaloids and biologically active compounds. ${ }^{7}$ Although many efforts have been made toward development of hydrogenation of quinolines using homogeneous achiral $\mathrm{Rh}^{8}$ or $\mathrm{Ru}^{9}$ catalysis, to the best of our knowledge, no report on homogeneous asymmetric hydrogenation of quinolines has appeared in the literature. Therefore, we decided to focus on this reaction. In this communication, we report our preliminary results and describe the first example of highly enantioselective iridium-catalyzed hydrogenation of quinolines.

Considering that iridium has been successfully applied to asymmetric hydrogenation of imines and unfunctionalized olefins recently, ${ }_{1}^{1}$ we first examined the $[\operatorname{Ir}(\mathrm{COD}) \mathrm{Cl}]_{2} / \mathrm{MeO}-\mathrm{Biphep} / \mathrm{DCM}$ system for hydrogenation of 2-methylquinoline (1a) (Scheme 1). Unfortunately, it was found that the catalytic activity is very low, and only a trace amount of product with low ee was obtained when the reaction was carried out in methylene chloride at room temperature under $700 \mathrm{psi}$ of hydrogen for $18 \mathrm{~h}$. A number of literature reports have appeared documenting the dramatic impact of additives on catalytic turnover and enantioselectivity. ${ }^{10}$ Accordingly, we evaluated a number of additives, such as $\mathrm{I}_{2}{ }^{11} n-\mathrm{Bu}_{4} \mathrm{NI},{ }^{12,13}$ $\mathrm{BiI}_{3},{ }^{13}$ phthalimide, ${ }^{14}$ benzylamine, ${ }^{15}$ etc., in an attempt to promote reaction turnover. Gratifyingly, iodine proved to be the most efficient additive in this reaction. Chiral iridium complex prepared in situ from $[\mathrm{Ir}(\mathrm{COD}) \mathrm{Cl}]_{2}$ and chiral bisphosphine $(R)$-MeO-Biphep were employed as catalysts, and the reactions were carried out at room temperature under 700 psi of hydrogen with a substrate:[Ir]: ligand: $\mathrm{I}_{2}$ ratio of $100: 0.5: 1.1: 10$. Further studies showed that this reaction was strongly solvent-dependent. As shown in Table 1, the reactions in THF, benzene, toluene, $\mathrm{DCM}, \mathrm{ClCH}_{2} \mathrm{CH}_{2} \mathrm{Cl}, i$-PrOH
Scheme 1

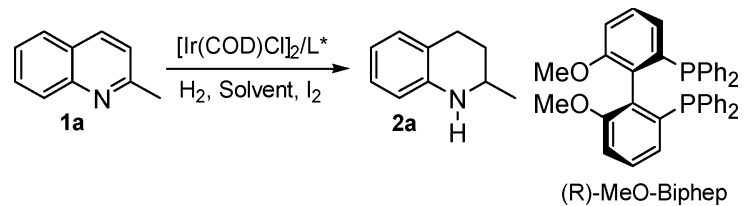

Table 1. Catalytic Asymmetric Hydrogenation of $1 \mathrm{a}^{a}$

\begin{tabular}{cclcc}
\hline entry & $\mathrm{H}_{2}(\mathrm{psi}) ; T\left({ }^{\circ} \mathrm{C}\right)$ & \multicolumn{1}{c}{ solvent } & convn $^{b}(\%)$ & $\mathrm{ee}^{c}(\%)$ \\
\hline 1 & $700 ; 25$ & $\mathrm{CH}_{2} \mathrm{Cl}_{2}$ & $>99$ & 85 \\
2 & $700 ; 25$ & $\left(\mathrm{ClCH}_{2}\right)_{2}$ & $>99$ & 85 \\
3 & $700 ; 25$ & $\mathrm{MeOH}$ & 85 & 9 \\
4 & $700 ; 25$ & $i-\mathrm{PrOH}$ & $>99$ & 84 \\
5 & $700 ; 25$ & THF & $>99$ & 85 \\
6 & $700 ; 25$ & toluene & $>99$ & 94 \\
7 & $700 ; 25$ & benzene & $>99$ & 94 \\
8 & $1500 ; 25$ & toluene & $>99$ & 91 \\
9 & $100 ; 25$ & toluene & 68 & 94 \\
10 & $700 ; 55$ & toluene & $>99$ & 90 \\
\hline
\end{tabular}

${ }^{a}$ Reaction conditions: $1 \mathrm{mmol}$ quinoline, $[\operatorname{Ir}(\mathrm{COD}) \mathrm{Cl}]_{2}(0.5 \%)$, chiral ligand $(1.1 \%), \mathrm{I}_{2}(10 \%), 5 \mathrm{~mL}$ solvent. ${ }^{b}$ Determined by ${ }^{1} \mathrm{H}$ NMR analysis of the crude products. ${ }^{c}$ Determined by HPLC analysis with Chiralpak OJ-H column, the absolute configuration of product is assigned by comparison of rotation sign with literature data.

could proceed well to give 2-methyl-1,2,3,4-tetrahydrohydroquinoline (2a) in excellent conversion in $18 \mathrm{~h}$ with high to excellent enantioselectivities. In contrast to alcoholic solvents (entries 3, 4), aprotic solvents (entries 1-2, 5-7) gave better enantioselectivities. In all cases, there were no 2-methyl-5,6,7,8-tetrahydroquinoline and 2-methyl-1,2,3,4,5,6,7,8-octahydro-quinoline detected by ${ }^{1} \mathrm{H}$ NMR analysis of the reaction mixture. A change in hydrogen pressure had no clear effect on enantioselectivity (entries 7-9), but conversion was decreased under lower pressure (entry 9). A slightly lower ee was obtained under higher temperature (entry 10). Therefore, the optimal condition for hydrogenation of $\mathbf{1 a}$ was shown in entry 6 . Under these experimental conditions, other commercially available chiral bidentate phosphine ligands were also tested for hydrogenation of $\mathbf{1 a}$, and it was found that enantioselectivities decreased whenever Ir-BINAP ( $87 \%$ ee), Ir-DIOP (53\% ee), or Ir$\mathrm{Me}-\mathrm{DuPhos}(51 \%$ ee) was used instead of Ir-MeO-Biphep (entry $6,94 \%$ ee). Thus, the optimized conditions are: $[\mathrm{Ir}(\mathrm{COD}) \mathrm{Cl}]_{2} / \mathrm{MeO}-$ Biphep/ $/ \mathrm{I}_{2} /$ toluene/ $/ \mathrm{H}_{2} 700 \mathrm{psi} / 25{ }^{\circ} \mathrm{C}$.

Under optimized condition, a variety of substituted quinoline derivatives were hydrogenated using $\mathrm{Ir} / \mathrm{MeO}-\mathrm{Biphep} / \mathrm{I}_{2}$ catalyst. Several 2-alkyl substituted quinolines were hydrogenated with high enantioselctivities ( $>92 \%$ ee) regardless of the length of side chain (entries 1-6 in Table 2). Branched substituent $(i-\operatorname{Pr})$ in the 2 position also gave good enantioselectivity (entry 18). 2-Arenethylsubstituted quinolines also gave excellent asymmetric induction (entries 7-9). With 2-aryl-substituted quinolines (entry 13), slightly lower enantioselectivity was obtained. It is noted that this catalytic system can tolerate hydroxyl and ester groups (entries 14-17, 19), 
Table 2. Iridium-Catalyzed Asymmetric Hydrogenation of $1^{a}$

\begin{tabular}{|c|c|c|c|}
\hline entry & R'/R of 1 & yield $^{b}$ & $\mathrm{Ee}^{\mathrm{c}}$ config $^{\mathrm{d}}$ \\
\hline 1 & $\mathrm{H} / \mathrm{Me}(\mathbf{1 a})$ & $94(\mathbf{2 a})$ & $94(\mathrm{R})$ \\
\hline 2 & $\mathrm{H} / \mathrm{Et}(\mathbf{1 b})$ & $88(2 b)$ & $96(\mathrm{R})$ \\
\hline 3 & $\mathrm{H} / n-\operatorname{Pr}(\mathbf{1 c})$ & $92(2 c)$ & $93(\mathrm{R})$ \\
\hline 4 & $\mathrm{H} / n-\mathrm{Bu}(\mathbf{1 d})$ & $86(2 d)$ & $92(\mathrm{R})$ \\
\hline 5 & H / 3-Butenyl (1e) & $91(\mathbf{2 d})^{\mathrm{e}}$ & $92(\mathrm{R})$ \\
\hline 6 & H / n-Pentyl (1f) & $92(\mathbf{2 f})$ & $94(\mathrm{R})$ \\
\hline 7 & H / Phenethyl (1g) & $94(2 \mathrm{~g})$ & $93(\mathrm{R})$ \\
\hline 8 & (1h) & $88(2 \mathrm{~h})$ & $93(\mathrm{R})$ \\
\hline 9 & $\mathrm{Me}$ & $86(2 i)$ & $96(\mathrm{R})$ \\
\hline 10 & $\mathrm{~F} / \mathrm{Me}(\mathbf{1} \mathbf{j})$ & $88(\mathbf{2 j})$ & $96(\mathrm{R})$ \\
\hline 11 & $\mathrm{Me} / \mathrm{Me}(\mathbf{1 k})$ & $91(\mathbf{2 k})$ & $91(\mathrm{R})$ \\
\hline 12 & $\mathrm{MeO} / \mathrm{Me}$ (11) & $89(\mathbf{2 l})$ & $84(\mathrm{R})$ \\
\hline 13 & $\mathrm{H} / \mathrm{Ph}(\mathbf{1 m})$ & $95(2 \mathrm{~m})$ & $72(\mathrm{~S})$ \\
\hline 14 & Me (1n) & $87(2 n)$ & $94(\mathrm{~S})$ \\
\hline 15 & & $89(2 p)$ & $92(\mathrm{~S})$ \\
\hline 16 & & $94(\mathbf{2 r})$ & $91(\mathrm{~S})$ \\
\hline 17 & $\mathrm{H} / \mathrm{CH}_{2} \mathrm{OH}(\mathbf{1 s})$ & $83(2 s)^{f}$ & $75(S)$ \\
\hline 18 & $\mathrm{H} / i-\operatorname{Pr}(\mathbf{1 t})$ & $92(\mathbf{2 t})$ & $94(\mathrm{~S})$ \\
\hline 19 & $\mathrm{H} / \mathrm{CH}_{2} \mathrm{OCOCH}_{3}(\mathbf{1} \mathbf{u})$ & $90(\mathbf{2 u})$ & $87(S)$ \\
\hline
\end{tabular}

${ }^{a}$ Reaction conditions: 1 mmol quinoline, $[\mathrm{Ir}(\mathrm{COD}) \mathrm{Cl}]_{2}(0.5 \%),(R)-\mathrm{MeO}-$ Biphep (1.1\%), $\mathrm{I}_{2}(10 \%), 5 \mathrm{~mL}$ toluene, $600-700 \mathrm{psi} \mathrm{H}_{2} .{ }^{b}$ Isolated yields based on quinolines. ${ }^{c}$ Determined by HPLC analysis. ${ }^{d}$ Determined by comparison of rotation sign with the literature data or by analogue. ${ }^{e}$ The product is $\mathbf{2 d}$. ${ }^{f} i$ - $\mathrm{PrOH}$ was used as solvent.

\section{Scheme 2}

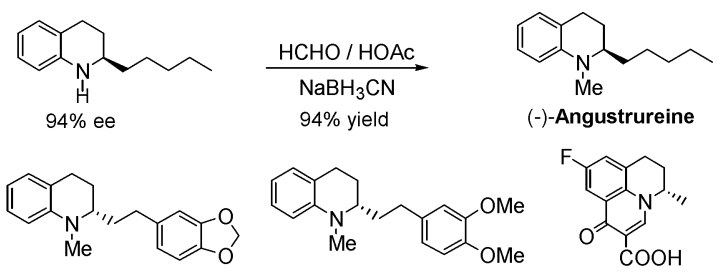

(-)-Galipinine, 94\% yield (-)-Cuspareine, $91 \%$ yield $\quad$ (S)-Flumequine

substrates with free hydroxyl, $\mathbf{1 n}, \mathbf{1 p}, \mathbf{1 r}$, and $\mathbf{1 s}$, can be hydrogenated smoothly with high enantioselectivities. The $\mathrm{C}=\mathrm{C}$ double bond in the side chain of substrate 1e (entry 5 ) can be hydrogenated under standard conditions. It is noted that very low enantioselectivities were obtained for 3- or 4-substituted quinoline derivatives ( $0 \%$ and $1 \%$ ee for 3 - and 4-methylquinoline, respectively), and the reason is not clear.

Iridium-catalyzed asymmetric hydrogenation of quinolines provides a convenient route to synthesize optically active tetrahydroquinoline derivatives since quinolines are very cheap starting materials. This methodology can be successfully applied to asymmetric synthesis of tetrahedroquinoline alkaloids (Scheme 2). For example, $\mathbf{2} \mathbf{f}, \mathbf{2} \mathbf{h}$, and $\mathbf{2 i}$ are naturally occurring tetrahydroquinoline alkaloids, ${ }^{7 \mathrm{e}} \mathrm{N}$-methylation of $\mathbf{2} \mathbf{f}, \mathbf{2} \mathbf{h}$, and $\mathbf{2 i}$ gives naturally occurring tetrahydroquinoline alkaloids angustureine, ${ }^{7 \mathrm{~b}}$ galipinine, ${ }^{7 \mathrm{c}, 7 \mathrm{~d}}$ and cuspareine, ${ }^{7 \mathrm{~d}}$ respectively in high total yields, and absolute configurations of $(+)$-angustureine and $(-)$-galipinine can be assigned through our synthesis (for the detailed procedure, see Supporting Information). $\mathbf{2} \mathbf{j}$ is the key intermediate in the synthesis of antibacterial agent of flumequine, ${ }^{16}$ which was obtained through the resolution method.

In conclusion, we developed the first example of highly enantioselective hydrogenation of quinoline derivatives using an $I r /$ phosphine $I_{2}$ system and applied it to the asymmetric synthesis of three naturally occurring alkaloids angustureine, galipinine, and cuspareine. This method provides an efficient access to a variety of optically active tetrahydroquinolines with up to $96 \%$ ee. Since tetrahydroquinoline derivatives are important synthetic intermediates and biologically active compounds, the current method has a high potential for practical use in organic synthesis. Further work will be directed toward detailed study of the reaction mechanism and the development of the hydrogenation of a broader range of substrates and other heteroaromatic systems.

Acknowledgment. We are grateful for the financial support from Talent Scientist Program, the Chinese Academy of Sciences.

Supporting Information Available: Characterization data for all compounds and experimental procedures (PDF). This material is available free of charge via the Internet at http://pubs.acs.org.

\section{References}

(1) For reviews, see: (a) Ojima, I., Ed. Catalytic Asymmetric Synthesis; VCH Publishers: New York, 1999. (b) Noyori, R. Ed. Asymmetric Catalysi in Organic Synthesis; Wiley: New York, 1994. (c) Jacobsen, E. N., Pfaltz, A., Yamamoto, H., Eds. Comprehensive Asymmetric Catalysis; Springer: Berlin, 1999; Vol. 1.

(2) Bird, C. W. Tetrahedron Lett. 1992, 48, 335-340

(3) Kuwano, R.; Sato, K.; Kurokawa, T.; Karube, D.; Ito, Y. J. Am. Chem Soc. 2000, 122, 7614-7615

(4) (a) Bianchini, C.; Barbaro, P.; Scapacci, G.; Farnetti, E.; Graziani, M. Organometallics 1998, 17, 3308-3310. (b) Bianchini, C.; Barbaro, P.; Scapacci, G. J. Organomet. Chem. 2001, 621, 26-33.

(5) (a) Studer, M.; Wedemeyer-Exl, C.; Spindler, F.; Blaser, H. U. Monatsh. Chem. 2002, 131, 1335. (b) Murata, S.; Sugimoto, T.; Matsuura, S. Heterocycles 1987, 26, 763-766.

(6) Keay, J. D. In Comprehensive Organic Synthesis; Trost, B. M., Fleming, I., Eds.; Pergamon: Oxford, 1991; Vol. 8, pp 579-601.

(7) (a) Barton, D. H. R.; Nakanishi, K.; Meth-Cohn, O., Eds. Comprehensive Natural Products Chemistry; Elsevier: Oxford, 1999; Vol. 1-9. (b) Jacquemond-Collet, I.; Hannedouche, S.; Fabre, N.; Fouraste, I.; Moulis, C. Phytochemistry 1999, 51, 1167-1169. (c) Rokotoson, J. H.; Fabre, N.; Jacquemond-Collet, I.; Hannedouche, S.; Fabre, N.; Fouraste, I.; Moulis, C. Planta Med. 1998, 64, 762-763. (d) Houghton, P. J.; Woldemariam, T. Z.; Watanabe, Y.; Yates, M. Planta Med. 1999, 65, 250-254. (e) Jacquemond-Collet, I.; Bessiere, J. M.; Hannedouche, S. Bertrand, C.; Fouraste, I.; Moulis, C. Phytochem. Anal. 2001, 12, 312319 .

(8) (a) Kuwano, R.; Sato, K.; Ito, Y. Chem. Lett. 2000, 428-430. (b) Baralt, E.; Smith, S. J.; Hurwitz, J.; Horvath, I. T.; Fish, R. H. J. Am. Chem Soc. 1992, 114, 5187-5196. (c) Fish, R. H.; Baralt, E.; Smith, S. J. Organometallics 1991, 10, 54-56. (d) Fish, R. H.; Tan, J. L.; Thormodsen, A. D. J. Org. Chem. 1984, 49, 4500-4505. (e) Murahashi, I.; Imada, Y.; Hirai, Y. Tetrahedron Lett. 1987, 28, 77-80. (f) Bianchini, C.; Barbaro, P.; Macchi, M.; Meli, A.; Vizza, F. Helv. Chim. Acta 2001, 84, 28952923

(9) (a) Bianchini, C.; Meli, A.; Moneti, S.; Oberhauser, W.; Vizza, F.; Herrera, V.; Fuentes, A.; Sauchez-Delgado, R. J. Am. Chem. Soc. 1999, 121, 70717080. (b) Fish, R. H.; Tan, J. L.; Thormodsen, A. D. Organometallics 1985, 4, 1743-1747 (c) Watanable, Y.; Ohta, T.; Tsuji, Y.; Hiyoshi, T. Tsuji, Y. Bull. Chem. Soc. Jpn. 1984, 57, 2440-2449.

(10) Vogl, E. M.; Groger, H.; Shibasaki, M. Angew. Chem., Int. Ed. 1999, 38 , $1570-1577$.

(11) (a) Togni, A. Angew. Chem., Int. Ed. Engl. 1996, 35, 1475-1477. (b) Xiao, D.; Zhang, X. Angew. Chem., Int. Ed. 2001, 40, 3425-3428.

(12) Morimoto, T.; Nakajima, N.; Achiwa, K. Chem. Pharm. Bull. 1994, 42, $1951-1953$.

(13) Morimoto, T.; Nakajima, N.; Achiwa, K. Synlett 1995, 748-750.

(14) (a) Zhu, G.; Zhang, X. Tetrahedron: Asymmetry 1998, 9, 2415-2418. (b) Morimoto, T.; Suzuki, N.; Achiwa, K. Heterocycles 1996, 43, 25572560 .

(15) Tani, K.; Onouchi, J.; Yamagata, T.; Kataoka, Y. Chem. Lett. 1995, 955956

(16) Balint, J.; Egri, G.; Fogassy, E., Bocskei, Z.; Simon, K.; Gajary, A.; Friesz, A. Tetrahedron: Asymmetry 1999, 10, 1079.

JA0353762 